\title{
Modafinil Effects on Middle-Frequency Oscillatory Power During Rule Selection in Schizophrenia
}

\author{
Michael J Minzenberg*,', Jong H Yoon ${ }^{2}$, Yaoan Cheng' and Cameron S Carter ${ }^{3,4}$ \\ 'Department of Psychiatry, University of California, San Francisco School of Medicine, and San Francisco Veterans Affairs Medical Center, \\ San Francisco, CA, USA; 'Department of Psychiatry, Stanford University School of Medicine, and Palo Alto Veterans Affairs Medical Center, Palo \\ Alto, CA, USA; ${ }^{3}$ Department of Psychiatry, University of California, Davis School of Medicine, Sacramento, CA, USA; ${ }^{4}$ Center for Neuroscience, \\ University of California, Davis, CA, USA
}

\begin{abstract}
Control-related cognitive processes such as rule selection are associated with cortical oscillations in the theta, alpha and, beta ranges, and modulated by catecholamine neurotransmission. Thus, a potential strategy for improving cognitive control deficits in schizophrenia would be to use pro-catecholamine pharmacological agents to augment these control-related oscillations. In a double-blind, placebo-controlled (within-subjects) study, we tested the effects of adjunctive single-dose modafinil $200 \mathrm{mg}$ on rule-related $4-30 \mathrm{~Hz}$ oscillations in 23 stable schizophrenia patients, using EEG during cognitive control task performance. EEG data underwent time-frequency decomposition with Morlet wavelets to determine the power of $4-30 \mathrm{~Hz}$ oscillations. Modafinil (relative to placebo) enhanced oscillatory power associated with high-control rule selection in theta, alpha, and beta ranges, with modest effects during rule maintenance. Modafinil treatment in schizophrenia augments middle-frequency cortical oscillatory power associated with rule selection, and may subserve diverse subcomponent processes in proactive cognitive control.

Neuropsychopharmacology (20I4) 39, 3018-3026; doi:I0.I038/npp.20I4.I55; published online 23 July 2014
\end{abstract}

\section{INTRODUCTION}

Cognitive control is supported by a distributed corticalsubcortical circuitry, with critical elements in the frontal cortex (Miller, 2000). This cognitive function and its neural basis are disturbed in schizophrenia (eg, Lesh et al, 2011; Minzenberg et al, 2009). Rule selection and representation is a critical element of cognitive control, as it guides taskor context-appropriate responses to the environment (Bunge, 2004). Studies in monkeys have identified how cortical oscillations support rule-representation, including theta (Benchenane et al, 2010; Womelsdorf et al, 2010), alpha and, beta in both rule acquisition (Benchenane et al, 2011) and rule selection (Buschman et al, 2012).

Given the role of these oscillatory frequencies in component processes related to cognitive control, it is not surprising that these oscillations are impaired in schizophrenia. Although the higher frequency gamma-range oscillations have been more intensively studied in schizophrenia, including those explicitly associated with cognitive control (Cho et al, 2006; Minzenberg et al, 2010), there is also an emerging literature that demonstrates altered

*Correspondence: Dr MJ Minzenberg, Outpatient Mental Health Service, II6C, San Francisco Veterans Affairs Medical Center, 4I50 Clement Street, San Francisco, CA 94121, USA, Tel: + I 916 415 221 $4810 \times 6554$, Fax: + 19164157506921 ,

E-mail: Michael.minzenberg@ucsf.edu

Received 12 March 20 14; revised 23 May 20I4; accepted 8 June 20I4; accepted article preview online 26 June 2014 oscillations in the lower frequencies. Using cognitive tasks that are highly-dependent on control processes, schizophrenia patients show reduced theta power (compared with matched healthy comparison groups) on Go/No-go tasks (Bates et al, 2009; Doege et al, 2010b), working memory encoding and retrieval (Haenschel et al, 2009; Schmiedt et al, 2005), and smooth-pursuit eye movement (Ivleva et al, 2013). Reduced theta power is also observed with auditory oddball task performance (Doege et al, 2009; Doege et al, 2010a). Schizophrenia patients also show reduced alpha power during encoding into working memory (Haenschel et al, 2009), and during Wisconsin Card Sort Test performance (Gonzalez-Hernandez et al, 2003), and reduced beta power with working memory encoding (Haenschel et al, 2009). Impairments in task-related oscillatory phaselocking have also been observed, including with beta oscillations during gestalt visual perception (Uhlhaas et al, 2006), and with theta and alpha in response to auditory click-trains (Brockhaus-Dumke et al, 2008). There is some variation in this literature, with one report of relatively increased resting and sensory-gated theta power (Hong et al, 2012), and auditory steady-state theta responses reported as increased (Kirihara et al, 2012) vs decreased (Hamm et al, 2011).

Control processes and cortical oscillations are also modulated by various neurochemical systems, suggesting their utility as drug targets in schizophrenia. Central catecholamine neurotransmitter systems arising from the locus coeruleus NE system (LC-NE) and the mesocortical dopamine (DA) 
system modulate complex cognitive functions such as working memory and cognitive control (Aston-Jones and Cohen, 2005; Durstewitz et al, 2000). The computational mechanism for these effects is enhanced gain in input/ output relationships of both individual neurons and neuronal populations. Similarly, brain oscillations are an important mechanism of gain control (Salinas and Sejnowski, 2001). The LC-NE densely innervates the midline frontal cortex (Steketee, 2003), which may be an independent generator of theta oscillations associated with various executive functions (Mitchell et al, 2008), and the parietal lobe is also very densely innervated by projections from the LC (Berridge and Waterhouse, 2003). These anatomic features of LC projections suggest that cognitive processes mediated by fronto-parietal networks may be particularly strongly modulated by this neurochemical system.

In an earlier fMRI study, we reported that modafinil, a low-potency inhibitor of the plasma membrane transporters for NE and DA (NET and DAT, respectively) (Madras et al, 2006; Volkow et al, 2009), enhances cognitive controlrelated activity in the LC, the distributed cognitive control network, and functional connectivity between the two (Minzenberg et al, 2008b). This was particularly observed for selection of high-control rules. These effects may form the basis of modafinil's enhancement of a range of controldependent cognitive processes in healthy and clinical populations (reviewed in Minzenberg and Carter, 2008a). We also recently reported that single-dose modafinil augments oscillatory power in the theta, alpha, and beta ranges in healthy subjects (Minzenberg et al, 2014) with high-control rule selection (relative to low-control rule selection), which is operationalized by presenting a visual cue that instructs an action to overcome a prepotent response tendency. To date, the effects of modafinil on brain oscillations related to cognition in schizophrenia patients have not been investigated. Indeed, only a single report is available addressing any pharmacological effects on task-related brain oscillations in schizophrenia. This study found that a drug with partial agonist activity at the gamma amino-butyric acid A receptor subtype increased control-related gamma oscillations (Lewis et al, 2008a). In light of the foregoing literature linking these oscillations to PFC-dependent cognitive processes, and the role of catecholamine systems, we hypothesized that modafinil administration would enhance middle-frequency cortical oscillations during high-control rule selection in a sample of schizophrenia patients.

\section{MATERIALS AND METHODS}

\section{Participants}

The study was conducted at the University of CaliforniaDavis Medical Center from February 2007 to July 2010. The ClinicalTrials.gov identifier is NCT00423943. All procedures were approved by the UCD Institutional Review Board, and all subjects provided informed consent for all procedures and were compensated for participation. Subjects were all outpatients, recruited from the community and our research clinic at UCD, and were included if they were aged 18-50, and lacked the following history: neurological illness, including head injury with loss of consciousness, uncorrectable visual problems, or peripheral motor disturbance; full-scale IQ $<70$ (by Wechsler Abbreviated Scale of Intelligence: WASI); known intolerance to modafinil; active substance-related disorder within 6 months prior to study; significant uncontrolled medical illness. All patients were evaluated with a structured diagnostic interview, using the SCID-I with DSM-IV-TR criteria administered by trained, reliable raters with masters or doctoral-level clinical training, and all subjects were assigned a 295.X diagnosis. The major symptoms of schizophrenia were evaluated using the Scale for the Assessment of Positive Symptoms (SAPS) and the Scale for the Assessment of Negative Symptoms (SANS). All subjects were free of illicit substances (determined by urine drug screening) at study. The demographic characteristics of the sample were as follows: mean age, $23.9 \pm 5.4$ years; percent male, $78 \%$ (18/23); mean personal education, $13.2 \pm 1.6$ years; mean parental education, $15.4 \pm 1.8$ years; mean fullscale IQ (WASI), $105 \pm 13$; mean illness duration (from onset of first psychotic episode), $3.7 \pm 4.9$ years; mean global SAPS score, $0.70 \pm 0.62$; mean global SANS score, 2.1 \pm 1.1 . All subjects were in active treatment with antipsychotic medication, primarily atypical antipsychotics, with a mean dose equal to $7.8 \pm 4.4$ haloperidol equivalents (Andreasen et al, 2010).

\section{Overview of Treatment and Testing Procedure}

Randomization of treatment order was performed without stratification, with a computer algorithm by a research pharmacist, who also packaged active medication and placebo in identical-appearing capsules for administration, and was otherwise uninvolved in the study. On a given test day, subjects were administered the drug or an identicallyappearing placebo in mid-morning. Subjects then waited in a quiet room for $1 \mathrm{~h}$ before the EEG preparation procedure, and initiated the cognitive task at $\sim 2 \mathrm{~h}$ after dosing, within the time span of peak circulating levels of modafinil (Robertson and Hellriegel, 2003). Subjects completed the test session and then returned after at least a 2-day interval to ensure washout of the study drug. Eleven subjects (48\%) completed the active drug testing day first, and the other twelve subjects completed the placebo day first.

\section{Cognitive Paradigm}

The cognitive task was presented using E-Prime (Psychological Software Tools, Pittsburgh, PA). EEG data were acquired during the Preparing to Overcome Prepotency task (Minzenberg et al, 2008b), a variant of a Simon spatialincompatibility task. The trial structure was as follows: cue (a green or red square), delay period, probe (a centrally presented white arrow pointing left or right, randomized with equal frequency between right and left directions), and a variable inter-trial interval (continuously varied between 1500 and $2500 \mathrm{~ms}$, from probe-onset to cue-onset of the subsequent trial). Both cue and probe stimuli had durations of $500 \mathrm{~ms}$. The cue-probe delay period (from cue-off to probe-on) was fixed at $1000 \mathrm{~ms}$, during which subjects were required to maintain fixation on a central fixation cross. Over this delay, subjects were required to maintain the appropriate rule (represented by the cue) to guide stimulusresponse (S-R) mappings to the probe. For the low-control condition (green-cued trials), subjects were required to 
respond with a button-press in the congruent direction of the subsequent arrow (eg, for a right-pointing arrow, press the right button, and left for left). For the high-control condition (red-cued trials, $45 \%$ of total), subjects responded in the incongruent direction (eg, for a right-pointing arrow, press the left button, and vice versa). Participants received eight blocks of 80 trials each, after one block of practice, which all subjects completed with errors on no more than two successive trials, as the criterion for proceeding to the experiment.

\section{Electroencephalography}

Data acquisition and offline processing. EEG data were acquired in a shielded room using a Neuroscan 128-electrode Quik-Cap and Neuroscan SynAmps2 hardware, with a sampling rate of $1000 \mathrm{~Hz}$ and a $100 \mathrm{~Hz}$ low-pass hardware filter. Data were collected using 32-bit encoding software, eliminating the need for high-pass recording filters. Electrode impedances were kept at $<5 \mathrm{k} \Omega$. All channels were referenced on line to $\mathrm{Cz}$. Malfunctioning electrodes were determined and excluded based on visual inspection of the impendence map and recorded waveforms. Data were then imported into EEGLab (Delorme and Makeig, 2004), re-referenced against the average reference, downsampled to $250 \mathrm{~Hz}$, and high-pass filtered at $0.5 \mathrm{~Hz}$. Epochs were extracted from the continuous EEG data, from -400 to +1700 ms relative to cue-onset. Each epoch was baselinecorrected using the pre-stimulus interval ( -400 to $0 \mathrm{~ms}$ ) in order to account for possible stimulus-independent ('background') fluctuations. Trials with incorrect responses were removed. Artifact rejection was performed with a probability-based criterion: First, the distribution of voltages averaged across all electrodes for a given trial was compared with the voltage for each individual electrode on that trial. If the individual electrode's voltage within that trial was $>5$ SDs from the mean of all electrodes, then the electrode was removed from that trial. A problematic case could occur if numerous electrodes exhibited excessive noise on a given trial, thereby making it difficult to discriminate an individual electrode's degree of noise from the full electrode set. However, this special case could be detected and resolved with the use of Independent Components Analysis (ICA), which followed this artifact rejection step (Onton and Makeig, 2006). This was performed using the 'logistic infomax' ICA algorithm (Bell and Sejnowski, 1995) with the 'extended' option (Lee et al, 1999); both available within EEGLab. Seventy-five principal components accounting for the most variance in the signal were derived, and of those, the top 15 components were identified for visualization and analysis. We used the methods of (McMenamin et al, 2010) and (Keren et al, 2010) to reject components in a principled manner, as follows. Upon obtaining these independent components, those suggestive of ocular artifacts (primarily eyeblinks, but also saccade-related components), muscle noise and other nonneural sources were identified via visual inspection of the equipotential scalp topography maps, the component waveforms, and the component time-frequency distributions, and comparison of each with the data available in (McMenamin et al, 2010) and (Keren et al, 2010). Eyeblink components were determined by their presence and proximity to the ocular area of the topography map, and their distinct waveform and time-frequency characteristics. Muscle noise components were determined primarily by their high frequency character.

Time-frequency transformation of the data. Time-frequency transformation of the data was performed using EEGLab (Delorme and Makeig, 2004), by convolving the epoched EEG with a complex Morlet wavelet function. These were performed on individual trial segments to identify timefrequency components in the desired ranges. one hertz-wide frequency sub-bands between $4-30 \mathrm{~Hz}$ were calculated separately, with each sub-band defined by a logarithmically increasing central frequency, and a range subject to a Gaussian kernel defined by the constant $c$, which is the ratio of the central frequency to the SD. For instance, time-frequency decomposition of the theta band $(4.55-8.36 \mathrm{~Hz})$ was performed with $\mathrm{c}=4$, and the period from -200 to $0 \mathrm{~ms}$ relative to cue-onset was defined as a baseline; average theta power during the baseline period was subtracted from task-related theta power determined during the trial. Time-frequency spectrograms were then established by pooling oscillatory power across electrodes grouped in topographically-organized subgroups as follows: Frontal, 9-13, 27-34, 53-61, 79-83, 8689, 106-113, 123; Parietal, 3-8, 14-16, 24-26, 35-37, 49-52, 62$64,75-78, \quad 90-92,103-105,114-116,120-122,126-128$; Occipital, 17-23, 38-48, 65-74, 93-102, 117-119.

Permutation method to empirically derive statistical thresholds. We sought to derive statistical thresholds appropriate for this data set, and importantly, to support statistical inferences made directly upon visual observation of spectrograms, in order to maximize the utility of timefrequency information available in these spectrograms. This allowed us to preserve the frequency and time-specificity of treatment effects, compared with binning power in increments of time or frequency. We first pooled (for each subject) the trial-averaged time-frequency wavelet coefficients into three electrode subgroups of approximately equal numbers ( $\sim 40$ electrodes in each subgroup), identified as Frontal, Parietal and Occipital subgroups. We then used a permutation method implemented in MatLab (Blair and Karniski, 1993). The procedure involved the following steps applied to the trial-averaged power values for the (red cue minus green cue) difference scores on Placebo vs Modafinil. First, we randomly switched the grouping of pairs of values from the two treatment conditions (to retain the paired nature of the statistical test), then repeated this for each of the remaining pairs in the conditions, and calculated the $t$ statistics for each pseudo-condition. This procedure was then repeated 4000000 times (to approximate the number of all possible combinations for this data set) to generate a distribution of $t$ statistics. We then compared the $t$ statistic observed in the comparison of each original time-frequency value between treatment conditions with this generated distribution, and determined the probability of this $t$-value against the distribution. The observed $t$-value is considered statistically significant if it is either less than half of the alpha value (ie, $p<0.025$ ) or greater than one minus half the alpha value (ie, $p>0.975$ ). Only these values are depicted as color-coded $t$-values in the spectrograms (see Results below). 


\section{RESULTS}

\section{Cognitive Task Performance}

The group means $( \pm S D)$ for each condition were as follows: Placebo Green Cue accuracy, $89.4 \pm 11.1 \%$; Placebo Red Cue accuracy, $88.0 \pm 12.4 \%$; Drug Green Cue accuracy, $90.0 \pm$ 10.4\%; Drug Red Cue accuracy, $88.7 \pm 11.1 \%$; Placebo Green Cue RT, $470 \pm 110 \mathrm{~ms}$; Placebo Red Cue RT, $479 \pm 98 \mathrm{~ms}$; Drug Green Cue RT, $481 \pm 123 \mathrm{~ms}$; Drug Red Cue RT, $493 \pm 120 \mathrm{~ms}$.

In an ANOVA of task accuracy, there was a significant main effect of cue $(\mathrm{F}=10.5$, df $1,22, p=0.004)$ but no significant effects of treatment $(\mathrm{F}=0.44, \mathrm{df}=1,22, p=0.52)$ or the treatment-by-cue interaction $(\mathrm{F}=0.01, \mathrm{df}=1,22$, $p=0.92)$. In ANOVA of RT, there was a significant main effect of cue $(\mathrm{F}=4.50, \mathrm{df}=1,22, p=0.045)$ but no significant effect of treatment $(\mathrm{F}=0.68, \mathrm{df}=1,22, p=0.42)$ or the treatment-by-cue interaction $(\mathrm{F}=0.32, \mathrm{df}=1,22$, $p=0.58)$.

\section{EEG Results}

Statistical inferences regarding task and treatment effects on cortical oscillations are made by reference to empirically thresholded spectrograms that depict (in parallel) oscillatory power in electrode subgroups (Figure 1), each throughout the theta to beta range $(4-30 \mathrm{~Hz})$ over the course of cue and cue-probe delay periods. As can be observed in Figure 1 top row, oscillatory power was roughly comparable (not significantly different) in response to highcontrol (red cue) vs low-control (green cue) trials on placebo (top row, 'Rule Selection on Placebo'), particularly with rule selection during the cue-on period. A notable exception was a relatively transient rule-related increase in the beta range in the middle of the delay period, observable in each electrode subgroup. In contrast, on modafinil (Figure 1 middle row), a relative increase in power for highcontrol vs low-control rule selection was observed in the theta, alpha, and beta ranges. This was observed in the beta ranges during cue-on period in the frontal and parietal electrode subgroups, primarily with a latency $\sim 200 \mathrm{~ms}$ after cue-onset, and relatively more dispersed over frequency and time in occipital electrodes, with theta, alpha, and beta ranges showing rule-related increases.

In direct comparison between modafinil and placebo (Figure 1 bottom row), drug treatment was associated with a significant relative increase in control-related oscillatory power ('Treatment-by-Rule Interaction') observed in the theta-alpha-beta range. In the frontal electrode subgroup, modafinil effects were primarily manifest in alpha and beta ranges at $\sim 100 \mathrm{~ms}$ and $200 \mathrm{~ms}$ after cue-onset. In the parietal electrode subgroup, modafinil effects were primarily evident in the beta range, at $\sim 200$ and $400 \mathrm{~ms}$ after cueonset. And in the occipital electrode subgroup, modafinil effects were again more dispersed in frequency and time, with the largest drug effects manifest in the theta range at $\sim 150 \mathrm{~ms}$, and beta-range effects at 200 and $400 \mathrm{~ms}$ after cue-onset. Significant differences in oscillatory power between the two treatment conditions were more variable during the delay period, though there was evidence of modest drug-related increases in the beta range scattered throughout the delay. The head-maps shown in Figure 2 illustrate the fronto-temporal topographic distribution of modafinil effects on control-related theta power, at $100 \mathrm{~ms}$ after cue-onset, when robust drug effects were observed in the full-head spectrogram. In contrast, in the beta range in the full-head spectrogram, the peak drug effects appear later within the cue-on period, and exhibit in addition to lateral frontal activity, a relatively more midline-parietal and occipital distribution, and are especially prominent in the late-delay period over the fronto-parietal scalp.

We also conducted a post hoc test of modafinil effects selectively on each rule (ie, cue) type, as a complementary approach to inform the inferential testing of treatment-byrule interactions. Here, we contrasted low-control (greencue)-related oscillatory power on modafinil $v s$ placebo, and in parallel, high-control (red-cue)-related oscillatory power on modafinil $v s$ placebo. The results (Figure 3 ) indicate that modafinil enhanced rule-related oscillatory power in theta, alpha, and beta ranges with high-control demands, in a manner not observed for low-control demands. These results are consistent with the inference that the observed drug effects on middle-frequency oscillations are controlrelated.

\section{DISCUSSION}

In the current study, we tested modafinil effects on middlefrequency oscillations in schizophrenia, in support of rule selection and representation as an aspect of cognitive control, which are important cognitive deficits observed in this illness. We found enhanced cue-period oscillatory power associated with high-control rule selection, in the theta, alpha, and beta ranges. These drug effects were notably more robust than the more transient, weaker, and more topographically variable drug effects on rule maintenance, observed during the delay period. The relative selectivity of modafinil effects on rule selection rather than maintenance is consistent with the findings from both our prior study of modafinil effects on middle-frequency oscillations during rule selection in healthy subjects (Minzenberg et al, 2014), as well as our fMRI study of healthy subjects, both of which used similar treatment procedures and cognitive paradigms as those used here (Minzenberg et al, 2008b).

Cue-period theta oscillatory power was strongly augmented by modafinil, whereas effects during the delay period were weaker and more transient, suggesting a specific drug effect on the selection of the rule, which guided the S-R mappings required as an expression of control in task performance. Sauseng et al (2010) suggest that cortical theta controls the access of information into working memory representations, consistent with the drug effect observed here. They and others have suggested that top-down control is achieved as a general feature in complex cognition via theta-mediated integration (Sauseng et al, 2010) and maintenance (Palva and Palva, 2007) of information in working memory, and alpha-mediated inhibition of taskirrelevant neuronal ensembles (Jensen and Mazaheri, 2010; Klimesch et al, 2007). These distinctions could explain the co-occurrence of drug effects on these adjacent frequency ranges during high-control rule selection in the present study. 

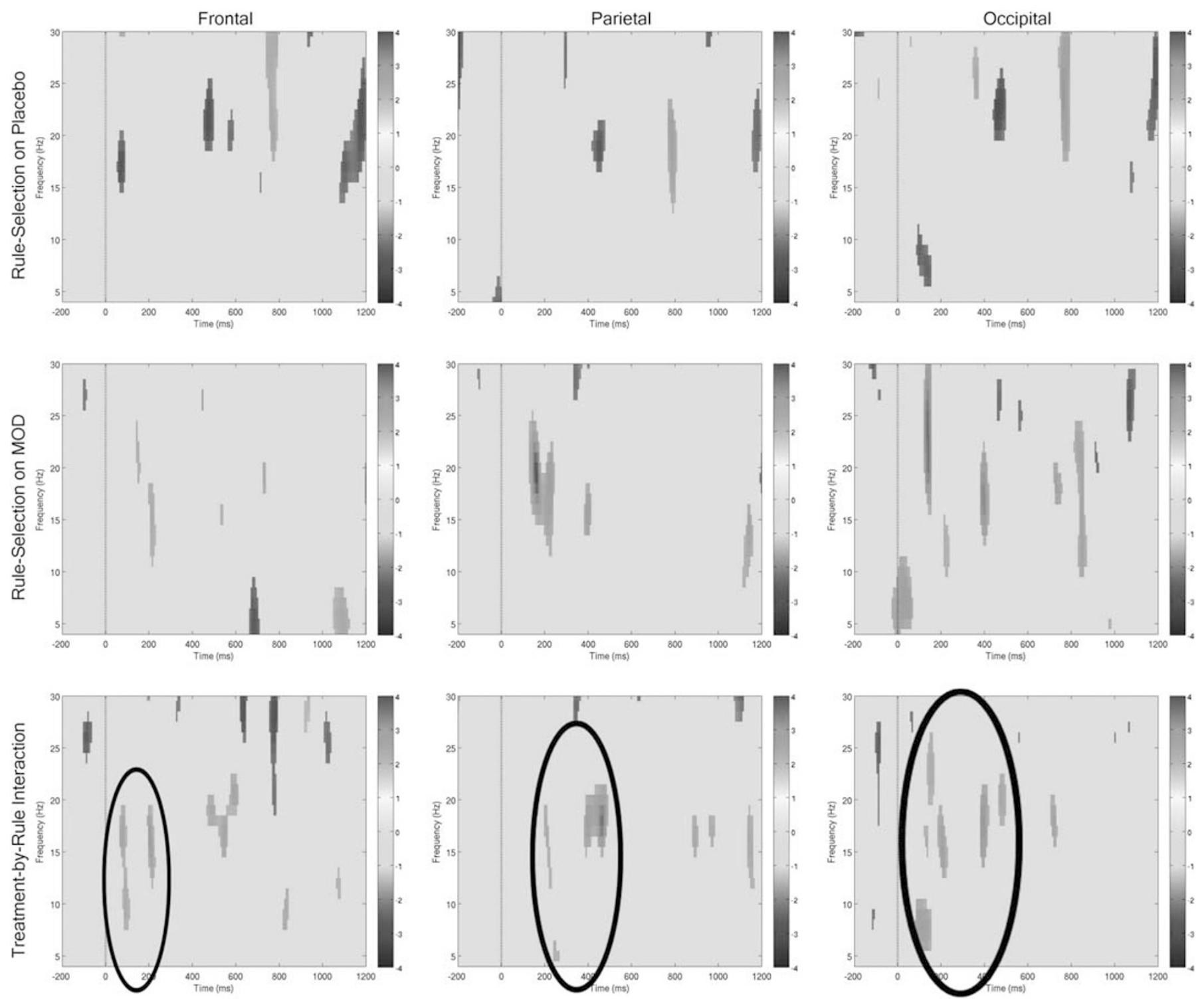

Figure I Spectrograms of task and drug effects on control-related middle-frequency power during proactive cognitive control task performance. Trial-averaged spectrograms depicting mean oscillatory power within 4-30 Hz range, from baseline period (pre-cue) through cue-on and cue-probe delay period, within electrode subgroups (indicated by headings). Vertical drop lines indicate the onset of the cue, at $t=0$; cue offset is at $t=500 \mathrm{~ms}$. Power is color-coded in all spectrograms according to scales at right, and color-coded only if exceeding the threshold derived from bootstrapping procedure (see text for details). Top row: oscillatory power in response to high-control (ie, Red Cue minus Green Cue) demands, on Placebo. Middle row: oscillatory power in response to high-control (ie, Red Cue minus Green Cue) demands, on modafinil. Bottom row: Modafinil effect on oscillatory power in response to highcontrol demands (ie, MOD (Red Cue minus Green Cue) minus PLC (Red Cue minus Green Cue)), or middle row minus top row. Note the robust relative increase in power in theta, alpha, and beta bands during the cue-on period. A full color version of this figure is available at Neuropsychopharmacology online.

Furthermore, a recent study of lateral PFC-mediated control processes in monkeys found beta-range oscillations associated with task-relevant rule selection, and alpha oscillations with the unselected rule (Buschman et al, 2012). In humans, beta oscillations are implicated in the control of both movement and cognition by 'endogenous', top-down processes that are usually synonymous with cognitive control (reviewed in Engel and Fries, 2010), and likely mediated via fronto-striatal circuitry (Jenkinson and Brown, 2011). Beta coherence is also increased in frontoparietal circuits in monkeys with demands for top-down control of attention (Buschman and Miller, 2007). Jenkinson and Brown (Jenkinson and Brown, 2011) suggest that beta oscillations in fronto-striatal circuits may specifically follow cues signaling that a prepared action must be suppressed, which characterizes the high-control task demand in the present study. These findings indicate roles for theta, alpha, and beta oscillations in PFC-mediated control processes, and the sensitivity of these task-related oscillations to modafinil suggests that catecholamine systems may modulate several subcomponent processes in cognitive control.

It remains unclear whether modafinil effects on cortical oscillations arise primarily from NE and/or DA systems in the brain. In our fMRI study, we found a complex pattern of 

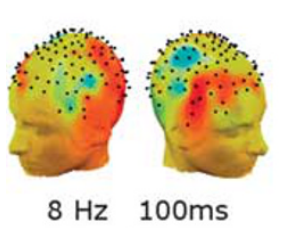

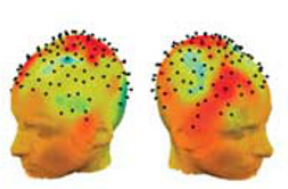

$15 \mathrm{~Hz} \quad 220 \mathrm{~ms}$

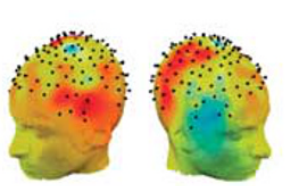

$18 \mathrm{~Hz} 480 \mathrm{~ms}$
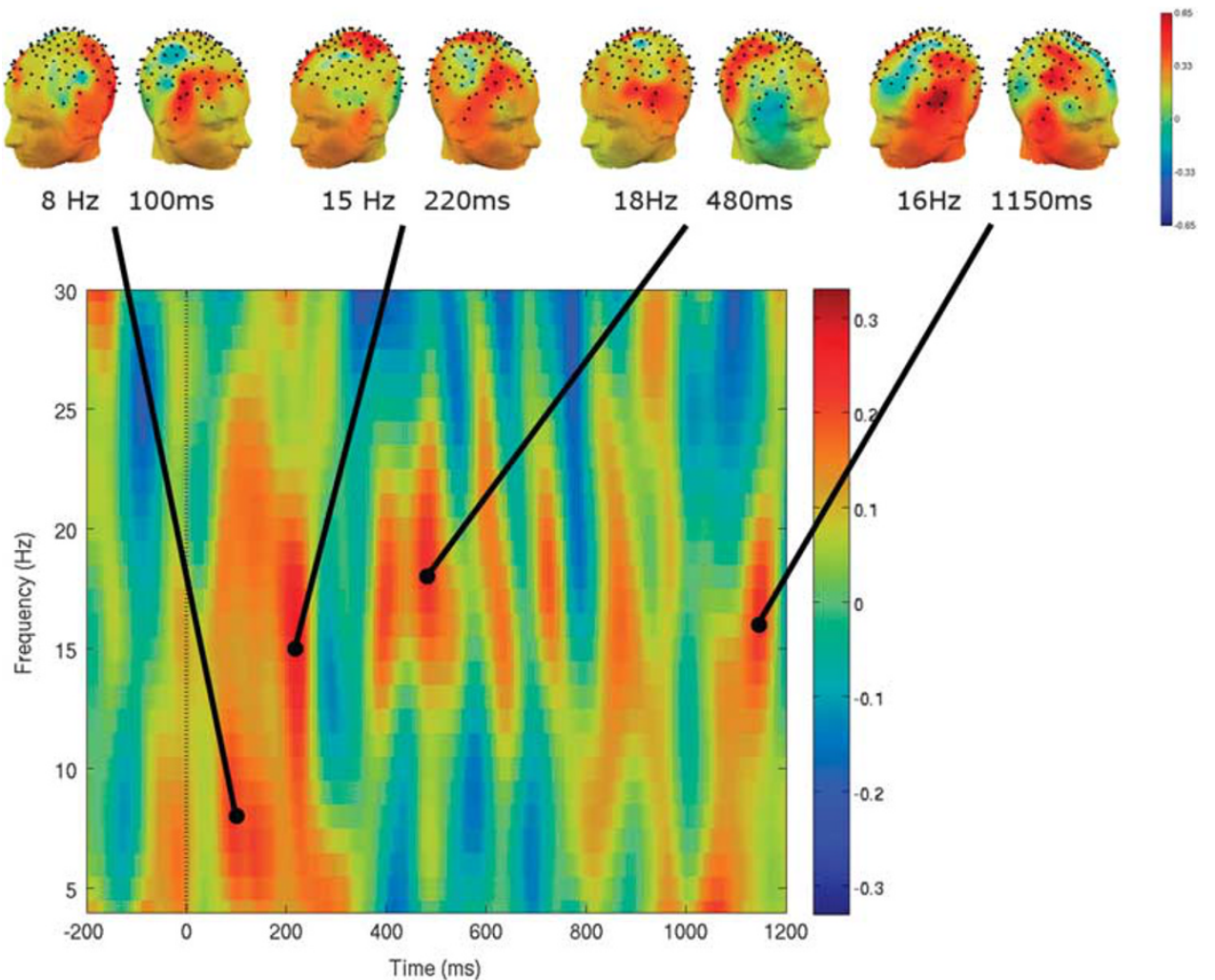

Figure 2 Topography of modafinil effects on control-related power in middle-frequencies. Group-averaged mean power as effect of drug-by-task interaction, across rule selection and maintenance task phases. Spectrogram depicts the mean power of all electrodes (statistical contrast as for Figure I bottom row) and head-maps depict scalp topography at time points of elevated power in theta and beta ranges. Note fronto-parietal distribution of drug effects during the cue-on period, with a different topography evident during delay-period power increases. Drop line at $t=0$ (cue-onset); $t=500 \mathrm{~ms}$ is cue offset.

modafinil effects on LC activation, and drug enhancement of control-related cortical activation and LC-PFC functional connectivity. These effects were highly consistent with a sophisticated model of the LC-NE system in optimizing cognitive processes associated with cortical function (Aston-Jones and Cohen, 2005). However, there is good evidence that DA neurotransmission in the PFC is also primarily regulated by the LC-NE system, because there is a paucity of DAT in the PFC, and therefore extracellular DA is primarily removed by NET activity (Carboni et al, 1990; Moron et al, 2002). It remains possible, therefore, that modafinil effects on LC activity are mediated by DA-receptor activation in terminal fields in the frontal cortex. Resolution of this issue may require drug-combination studies where the effects of catecholamine transporter inhibition are modified by specific catecholamine receptor ligands, such as beta-blockers or selective DA-receptor antagonists.

These findings may in fact stem from effects on both systems. Both NE and DA have direct influences on cortical pyramidal cells, via a diversity of receptors, including every major subtype among these two neurotransmitter systems, at pre- and postsynaptic sites ( $\mathrm{Gu}, 2002)$. In addition, catecholamines directly innervate cortical inhibitory interneurons, and catecholamine effects on cortical principal cells may be largely mediated via local inhibitory interneurons (Bacci et al, 2005). Norepinephrine depolarizes fast-spiking interneurons in rat frontal cortex, including chandelier cells (Kawaguchi and Shindou, 1998), has heterogeneous effects on CCK + interneurons (Kawaguchi and Shindou, 1998), and depolarizes hippocampal interneurons (Bergles et al, 1996). Similarly, DA increases the excitability of fast-spiking, non-adapting interneurons in primate DLPFC, including basket cells and chandelier cells (Kroner et al, 2007). The gating of pyramidal cell inputs and outputs by these interneurons are critical determinants of cortical oscillations, including those in theta, alpha, and beta frequency ranges (Freund, 2003; Gonzalez-Burgos and Lewis, 2008; Jensen and Mazaheri, 2010; Whittington and Traub, 2003). These cortical interneuron cell types, cellular and population-level physiological phenomena, have each been implicated in schizophrenia (GonzalezBurgos and Lewis, 2008). These observations suggest that catecholamine systems modulate cortical oscillations in task-relevant cortical ensembles, phenomena that are highly-relevant for the pathophysiology of schizophrenia, and therefore serve as candidate treatment targets.

Modafinil has also shown positive effects on EEG measures in other clinical populations, including in alpha and beta power (Saletu et al, 2004), and enhanced N1 in the left auditory cortex and for P300 in the medial and right dorsolateral prefrontal cortex (Saletu et al, 2009), both in narcolepsy patients; and in multiple sclerosis patients, pre-treatment auditory P300 latency predicted treatment 

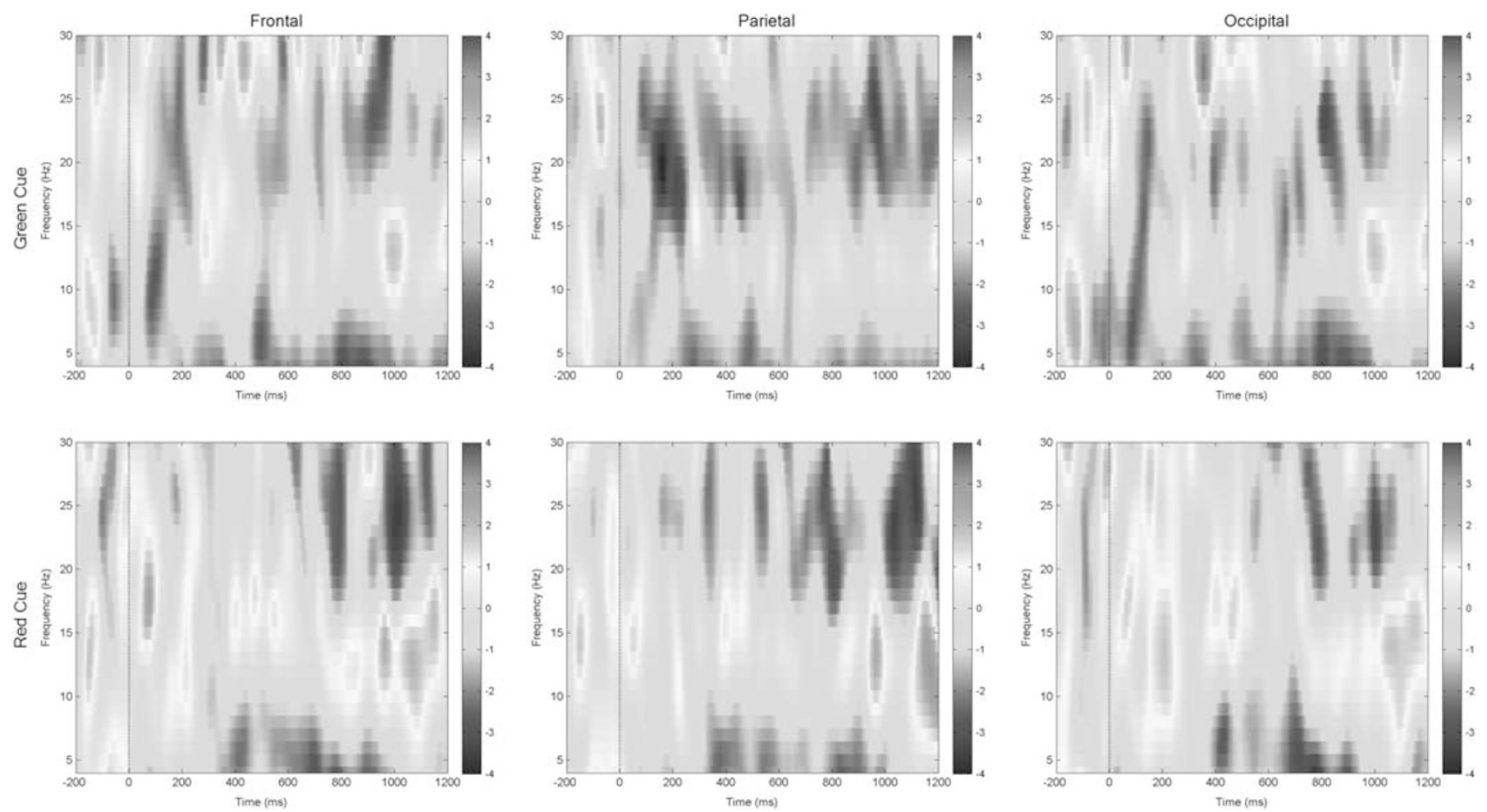

Figure 3 Spectrograms of drug effects on middle-frequency power specified by rule during proactive cognitive control task performance. Trial-averaged spectrograms depicting mean oscillatory power within $4-30 \mathrm{~Hz}$ range, from baseline period (pre-cue) through cue-on and cue-probe delay period, within electrode subgroups (indicated by headings). Vertical drop lines indicate the onset of the cue, at $t=0$; cue offset is at $t=500 \mathrm{~ms}$. Power is color-coded in all spectrograms according to scales at right. Top row: oscillatory power in response to low-control (ie, green cue) demands, on modafinil vs placebo. Bottom row: oscillatory power in response to high-control (ie, red cue) demands, on modafinil vs placebo. Note the robust relative increase in power in theta, alpha and beta bands during the cue-on period for high-control (red-cued) demands, on modafinil. A full color version of this figure is available at Neuropsychopharmacology online.

response to modafinil (Nagels et al, 2007); and after sleep deprivation in healthy subjects, modafinil partly normalized both the increased delta and theta activity, and the reduced alpha and beta activity, that were each observed on placebo (James et al, 2011).

In schizophrenia, there are also a number of clinical considerations with the potential use of modafinil as an adjunctive treatment. For instance, it remains unclear how the effects of single-doses of modafinil may relate to those with more sustained treatment regimens. Modafinil shares many cellular and clinical effects with classic stimulants, which remediate cognition in patients with attention-deficit disorder on a dose-by-dose basis (see review in Minzenberg and Carter, 2008a). Yet modafinil also shares effects on NET with many classic antidepressants, which have a wellestablished latency to therapeutic action, which is likely based on downstream, intracellular effects on second messengers, gene transcription, and possibly neurogenesis (Tanis and Duman, 2007). A note of caution is also warranted with the use of modafinil in schizophrenia. There are two potential sources of risk in this setting: (1) modafinil induces the CYP3A4 isozyme, which contributes to degradation of many atypical antipsychotics (Robertson and Hellriegel, 2003), therefore modafinil administration could lead to reduced bioavailability of concurrent antipsychotics; (2) modafinil as a weak stimulant could theoretically provoke psychosis. Fortunately, the empirical evidence available to date suggests at most a small risk of provoking psychosis with the use of modafinil in nonpsychotic (Davies et al, 2013) or schizophrenia (SaavedraVelez et al, 2009) patients. It also remains unclear if pharmacodynamic interactions between modafinil and antipsychotics may limit the efficacy of the former. Antipsychotics (as a rule) have significant DA antagonism, and many have significant anti-adrenergic effects as well (Minzenberg and Yoon, 2011). These effects may mitigate the benefit of pro-catecholaminergic agents that might otherwise effectively modulate these neurotransmitter systems. Future work should address these issues in order to advance both clinical knowledge and models of brain function that can support the development of novel pro-cognitive agents.

\section{CONCLUSION}

Middle-frequency oscillations are associated with several important component processes of cognitive control, and these oscillations and their cognitive correlates are impaired in schizophrenia. This suggests that these physiological phenomena may be important candidate treatment targets in this illness. Modafinil is a novel drug that augments signaling in catecholamine systems that in turn modulate these neuronal systems and cortical phenomena, and prior work has indicated positive effects of modafinil on these 
processes in healthy subjects. The present results indicate that these effects can be observed as well in stable, medicated schizophrenia patients. Future work should further elaborate on mechanisms of action in catecholamine modulation of these physiological phenomena, and address the optimal conditions for remediation of these deficits in schizophrenia, including treatment considerations such as optimal dose and duration of treatment, potential interactions with other existing and potential treatments, and the clinical and functional consequences for schizophrenia patients.

\section{FUNDING AND DISCLOSURE}

This work was supported by a Clinical Scientist Development Award from the Doris Duke Charitable Foundation, and a Young Investigator award from NARSAD (Brain and Behavior Foundation), both to MJM, and 5RO1 MH059883 to CSC. The authors have no competing financial interests.

\section{ACKNOWLEDGEMENTS}

We acknowledge the assistance of Glenn Gomes, Janeth Nunez del Prado, Jenny Yen and Dennis Thompson in the conduct and analysis of the study.

\section{REFERENCES}

Andreasen NC, Pressler M, Nopoulos P, Miller D, Ho BC (2010). Antipsychotic dose equivalents and dose-years: a standardized method for comparing exposure to different drugs. Biol Psychiatry 67: 255-262.

Aston-Jones G, Cohen JD (2005). An integrative theory of locus coeruleus-norepinephrine function: adaptive gain and optimal performance. Ann Rev Neurosci 28: 403-450.

Bacci A, Huguenard JR, Prince DA (2005). Modulation of neocortical interneurons: extrinsic influences and exercises in self-control. Trends Neurosci 28: 602-610.

Bates AT, Kiehl KA, Laurens KR, Liddle PF (2009). Low-frequency EEG oscillations associated with information processing in schizophrenia. Schizophr Res 115: 222-230.

Bell AJ, Sejnowski TJ (1995). An information-maximization approach to blind separation and blind deconvolution. Neural Comput 7: 1129-1159.

Benchenane K, Peyrache A, Khamassi M, Tierney PL, Gioanni Y, Battaglia FP et al (2010). Coherent theta oscillations and reorganization of spike timing in the hippocampal- prefrontal network upon learning. Neuron 66: 921-936.

Benchenane K, Tiesinga PH, Battaglia FP (2011). Oscillations in the prefrontal cortex: a gateway to memory and attention. Curr Opin Neurobiol 21: 475-485.

Bergles DE, Doze VA, Madison DV, Smith SJ (1996). Excitatory actions of norepinephrine on multiple classes of hippocampal CA1 interneurons. J Neurosci 16: 572-585.

Berridge CW, Waterhouse BD (2003). The locus coeruleusnoradrenergic system: modulation of behavioral state and state-dependent cognitive processes. Brain Res Rev 42: 33-84.

Blair RC, Karniski W (1993). An alternative method for significance testing of waveform difference potentials. Psychophysiology 30: 518-524.

Brockhaus-Dumke A, Mueller R, Faigle U, Klosterkoetter J (2008). Sensory gating revisited: relation between brain oscillations and auditory evoked potentials in schizophrenia. Schizophr Res 99: 238-249.
Bunge SA (2004). How we use rules to select actions: a review of evidence from cognitive neuroscience. Cogn Affect Behav Neurosci 4: 564-579.

Buschman TJ, Denovellis EL, Diogo C, Bullock D, Miller EK (2012). Synchronous oscillatory neural ensembles for rules in the prefrontal cortex. Neuron 76: 838-846.

Buschman TJ, Miller EK (2007). Top-down versus bottom-up control of attention in the prefrontal and posterior parietal cortices. Science 315: 1860-1862.

Carboni E, Tanda GL, Frau R, Di Chiara G (1990). Blockade of the noradrenaline carrier increases extracellular dopamine concentrations in the prefrontal cortex: evidence that dopamine is taken up in vivo by noradrenergic terminals. J Neurochem 55: 1067-1070.

Cho RY, Konecky RO, Carter CS (2006). Impairments in frontal cortical gamma synchrony and cognitive control in schizophrenia. Proc Natl Acad SciUSA 103: 19878-19883.

Davies M, Wilton L, Shakir S (2013). Safety profile of modafinil across a range of prescribing indications, including off-label use, in a primary care setting in England: results of a modified prescription-event monitoring study. Drug Saf 36: 237-246.

Delorme A, Makeig S (2004). EEGLAB: an open source toolbox for analysis of single-trial EEG dynamics including independent component analysis. J Neurosci Met 134: 9-21.

Doege K, Bates AT, White TP, Das D, Boks MP, Liddle PF (2009). Reduced event-related low frequency EEG activity in schizophrenia during an auditory oddball task. Psychophysiology 46: 566-577.

Doege K, Jansen M, Mallikarjun P, Liddle EB, Liddle PF (2010a). How much does phase resetting contribute to event-related EEG abnormalities in schizophrenia? Neurosci Lett 481: 1-5.

Doege K, Kumar M, Bates AT, Das D, Boks MP, Liddle PF (2010b). Time and frequency domain event-related electrical activity associated with response control in schizophrenia. Clin Neurophysiol 121: 1760-1771.

Durstewitz D, Seamans JK, Sejnowski TJ (2000). Neurocomputational models of working memory. Nat Neurosci 3 Suppl: 1184-1191.

Engel AK, Fries P (2010). Beta-band oscillations-signalling the status quo? Curr Opin Neurobiol 20: 156-165.

Freund TF (2003). Interneuron Diversity series: Rhythm and mood in perisomatic inhibition. Trends Neurosci 26: 489-495.

Gonzalez-Burgos G, Lewis DA (2008). GABA neurons and the mechanisms of network oscillations: implications for understanding cortical dysfunction in schizophrenia. Schizophr Bull 34: 944-961.

Gonzalez-Hernandez JA, Cedeno I, Pita-Alcorta C, Galan L, Aubert E, Figueredo-Rodriguez P (2003). Induced oscillations and the distributed cortical sources during the Wisconsin card sorting test performance in schizophrenic patients: new clues to neural connectivity. Int J Psychophysiol 48: 11-24.

$\mathrm{Gu} \mathrm{Q}$ (2002). Neuromodulatory transmitter systems in the cortex and their role in cortical plasticity. Neuroscience 111: 815-835.

Haenschel C, Bittner RA, Waltz J, Haertling F, Wibral M, Singer W et al (2009). Cortical oscillatory activity is critical for working memory as revealed by deficits in early-onset schizophrenia. J Neurosci 29: 9481-9489.

Hamm JP, Gilmore CS, Picchetti NA, Sponheim SR, Clementz BA (2011). Abnormalities of neuronal oscillations and temporal integration to low- and high-frequency auditory stimulation in schizophrenia. Biol Psychiatry 69: 989-996.

Hong LE, Summerfelt A, Mitchell BD, O’Donnell P, Thaker GK (2012). A shared low-frequency oscillatory rhythm abnormality in resting and sensory gating in schizophrenia. Clin Neurophysiol 123: 285-292.

Ivleva EI, Moates AF, Hamm JP, Bernstein IH, O'Neill HB, Cole D et al (2013). Smooth pursuit eye movement, prepulse inhibition, and auditory paired stimuli processing endophenotypes 
across the schizophrenia-bipolar disorder psychosis dimension. Schizophr Bull 40: 642-652.

James LM, Iannone R, Palcza J, Renger JJ, Calder N, Cerchio K et al (2011). Effect of a novel histamine subtype-3 receptor inverse agonist and modafinil on EEG power spectra during sleep deprivation and recovery sleep in male volunteers. Psychopharmacology 215: 643-653.

Jenkinson N, Brown P (2011). New insights into the relationship between dopamine, beta oscillations and motor function. Trends Neurosci 34: 611-618.

Jensen O, Mazaheri A (2010). Shaping functional architecture by oscillatory alpha activity: gating by inhibition. Front Hum Neurosci 4: 186.

Kawaguchi Y, Shindou T (1998). Noradrenergic excitation and inhibition of GABAergic cell types in rat frontal cortex. J Neurosci 18: 6963-6976.

Keren AS, Yuval-Greenberg S, Deouell LY (2010). Saccadic spike potentials in gamma-band EEG: characterization, detection and suppression. Neuroimage 49: 2248-2263.

Kirihara K, Rissling AJ, Swerdlow NR, Braff DL, Light GA (2012). Hierarchical organization of gamma and theta oscillatory dynamics in schizophrenia. Biol Psychiatry 71: 873-880.

Klimesch W, Sauseng P, Hanslmayr S (2007). EEG alpha oscillations: the inhibition-timing hypothesis. Brain Res Rev 53: 63-88.

Kroner S, Krimer LS, Lewis DA, Barrionuevo G (2007). Dopamine increases inhibition in the monkey dorsolateral prefrontal cortex through cell type-specific modulation of interneurons. Cereb Cortex 17: 1020-1032.

Lee TW, Girolami M, Sejnowski TJ (1999). Independent component analysis using an extended infomax algorithm for mixed subgaussian and supergaussian sources. Neural Comput 11: 417-441.

Lesh TA, Niendam TA, Minzenberg MJ, Carter CS (2011). Cognitive control deficits in schizophrenia: mechanisms and meaning. Neuropsychopharmacology 36: 316-338.

Lewis DA, Cho RY, Carter CS, Eklund K, Forster S, Kelly MA et al (2008a). Subunit-selective modulation of GABA type A receptor neurotransmission and cognition in schizophrenia. AmJ Psychiatry 165: 1585-1593.

Madras BK, Xie Z, Lin Z, Jassen A, Panas H, Lynch L et al (2006). Modafinil occupies dopamine and norepinephrine transporters in vivo and modulates the transporters and trace amine activity in vitro. J Pharmacol Exp Ther 319: 561-569.

McMenamin BW, Shackman AJ, Maxwell JS, Bachhuber DR, Koppenhaver AM, Greischar LL et al (2010). Validation of ICAbased myogenic artifact correction for scalp and sourcelocalized EEG. NeuroImage 49: 2416-2432.

Miller EK (2000). The prefrontal cortex and cognitive control. Nat Rev Neurosci 1: 59-65.

Minzenberg MJ, Carter CS (2008a). Modafinil: a review of neurochemical actions and effects on cognition. Neuropsychopharmacology 33: 1477-1502.

Minzenberg MJ, Firl AJ, Yoon JH, Gomes GC, Reinking C, Carter CS (2010). Gamma oscillatory power is impaired during cognitive control independent of medication status in first-episode schizophrenia. Neuropsychopharmacology 35: 2590-2599.

Minzenberg MJ, Gomes GC, Yoon JH, Watrous AJ, Geng JJ, Firl AJ et al (2014). Modafinil augments oscillatory power in middlefrequencies during rule selection. Psychophysiology 51: 510-519.

Minzenberg MJ, Laird AR, Thelen S, Carter CS, Glahn DC (2009). Meta-analysis of 41 functional neuroimaging studies of executive function in schizophrenia. Arch Gen Psychiatry 66: 811-822.

Minzenberg MJ, Watrous AJ, Yoon JH, Ursu S, Carter CS (2008b). Modafinil shifts human locus coeruleus to low-tonic, highphasic activity during functional MRI. Science 322: 1700-1702.
Minzenberg MJ, Yoon JH (2011). An index of relative central alpha-adrenergic receptor antagonism by antipsychotic medications. Exp Clin Psychopharmacol 19: 31-39.

Mitchell DJ, McNaughton N, Flanagan D, Kirk IJ (2008). Frontalmidline theta from the perspective of hippocampal 'theta'. Prog Neurobiol 86: 156-185.

Moron JA, Brockington A, Wise RA, Rocha BA, Hope BT (2002). Dopamine uptake through the norepinephrine transporter in brain regions with low levels of the dopamine transporter: evidence from knock-out mouse lines. J Neurosci 22: 389-395.

Nagels G, D'Hooghe M B, Vleugels L, Kos D, Despontin M, De Deyn PP (2007). P300 and treatment effect of modafinil on fatigue in multiple sclerosis. J Cli Neurosci 14: 33-40.

Onton J, Makeig S (2006). Information-based modeling of eventrelated brain dynamics. Prog Brain Res 159: 99-120.

Palva S, Palva JM (2007). New vistas for alpha-frequency band oscillations. Trends Neurosci 30: 150-158.

Robertson P Jr., Hellriegel ET (2003). Clinical pharmacokinetic profile of modafinil. Clin Pharmacokinet 42: 123-137.

Saavedra-Velez C, Yusim A, Anbarasan D, Lindenmayer JP (2009). Modafinil as an adjunctive treatment of sedation, negative symptoms, and cognition in schizophrenia: a critical review. J Clin Psychiatry 70: 104-112.

Saletu M, Anderer P, Saletu-Zyhlarz GM, Mandl M, Arnold O, Zeitlhofer J et al (2004). EEG-tomographic studies with LORETA on vigilance differences between narcolepsy patients and controls and subsequent double-blind, placebo-controlled studies with modafinil. J Neurol 251: 1354-1363.

Saletu M, Anderer P, Saletu-Zyhlarz GM, Mandl M, Saletu B, Zeitlhofer J (2009). Modafinil improves information processing speed and increases energetic resources for orientation of attention in narcoleptics: double-blind, placebo-controlled ERP studies with low-resolution brain electromagnetic tomography (LORETA). Sleep Med 10: 850-858.

Salinas E, Sejnowski TJ (2001). Gain modulation in the central nervous system: where behavior, neurophysiology, and computation meet. Neuroscientist 7: 430-440.

Sauseng P, Griesmayr B, Freunberger R, Klimesch W (2010). Control mechanisms in working memory: a possible function of EEG theta oscillations. Neurosci Biobehav Rev 34: 1015-1022.

Schmiedt C, Brand A, Hildebrandt H, Basar-Eroglu C (2005). Event-related theta oscillations during working memory tasks in patients with schizophrenia and healthy controls. Cognitive Brain Res 25: 936-947.

Steketee JD (2003). Neurotransmitter systems of the medial prefrontal cortex: potential role in sensitization to psychostimulants. Brain Res Rev 41: 203-228.

Tanis KQ, Duman RS (2007). Intracellular signaling pathways pave roads to recovery for mood disorders. Ann Med 39: 531-544.

Uhlhaas PJ, Linden DE, Singer W, Haenschel C, Lindner M, Maurer K et al (2006). Dysfunctional long-range coordination of neural activity during Gestalt perception in schizophrenia. J Neurosci 26: 8168-8175.

Volkow ND, Fowler JS, Logan J, Alexoff D, Zhu W, Telang F et al (2009). Effects of modafinil on dopamine and dopamine transporters in the male human brain: clinical implications. JAMA 301: 1148-1154.

Whittington MA, Traub RD (2003). Interneuron diversity series: inhibitory interneurons and network oscillations in vitro. Trends Neurosci 26: 676-682.

Womelsdorf T, Johnston K, Vinck M, Everling S (2010). Theta-activity in anterior cingulate cortex predicts task rules and their adjustments following errors. Proc Natl Acad Sci USA 107: 5248-5253. 